Van Binsbergen, Wim M. J., ed. -- « "Black Athena" : Ten Years After ». Talanta [Amsterdam], XXVIIIXXIX/1996-1997, 272 p., index, bibl.

\title{
Vittorio Morabito
}

\section{OpenEdition}

\section{Journals}

Édition électronique

URL : http://journals.openedition.org/etudesafricaines/183

DOI : 10.4000/etudesafricaines. 183

ISSN : 1777-5353

Éditeur

Éditions de l'EHESS

\section{Édition imprimée}

Date de publication : 1 janvier 2000

ISBN : 978-2-7132-1356-4

ISSN : 0008-0055

Référence électronique

Vittorio Morabito, « Van Binsbergen, Wim M. J., ed. -- «"Black Athena" : Ten Years After ». Talanta [Amsterdam], XXVIII-XXIX/1996-1997, 272 p., index, bibl. », Cahiers d'études africaines [En ligne], 158 2000, mis en ligne le 02 mai 2003, consulté le 05 février 2021. URL : http://journals.openedition.org/ etudesafricaines/183 ; DOI : https://doi.org/10.4000/etudesafricaines.183

Ce document a été généré automatiquement le 5 février 2021.

(c) Cahiers d'Études africaines 


\title{
Van Binsbergen, Wim M. J., ed. -- «"Black Athena" : Ten Years After ». Talanta [Amsterdam], XXVIII-XXIX/1996-1997, 272 p., index, bibl.
}

\author{
Vittorio Morabito
}

Les sujets traités dans cet ouvrage prolongent le débat, tenu dans Black Athena Revisited ${ }^{1}$, qui réunit quelques grands noms de "spécialistes ", lesquels récusent la vision de Martin Bernal, l'auteur de Black Athena $[B A]^{2}$ et le « perturbateur » de l'ordre établi dans les études afro-asiatiques sur l'antiquité. Les Actes de la Journée d'études, organisée aux Pays-Bas le 28 juin 1996 avec le concours du Centre d'études africaines de Leiden, sont publiés dans un numéro double de la revue d'archéologie Talanta. Un index général, fort exhaustif, relatif aux sujets et aux noms propres évoqués, enrichit le volume. M. Bernal collabore aux neuf interventions et apporte trois Réponses aux conférenciers, toutes centrées sur l'interprétation incontestable de sa pensée. Elle a pourtant déjà sérieusement été passée au crible par des dizaines de critiques, surtout dans le monde anglo-saxon, et a été défendue par des groupes africains américains. L'intérêt soulevé par $B A$ n'est pas seulement dû à la reprise de la thèse historique qui place l'Afrique à l'origine des cultures égyptienne et grecque, ou aux accusations lancées contre ce discours, mais l'intérêt porte également sur l'ouverture à de nouvelles perspectives de recherches « scientifiques » et à des problèmes d'actualité, comme la quête d'identité des Noirs américains et le rôle joué par la culture juive. L'origine de la civilisation grecque, selon M. Bernal, remonte à la fois à l'Égypte pharaonique et aux populations sémitiques, en particulier aux Phéniciens. La civilisation égyptienne, de son côté, doit son existence à la propagation de la civilisation afro-asiatique exprimée dans le titre « Athéna noire ». Les Grecs euxmêmes, Hérodote et Eschyle en premier, et les chercheurs les plus éminents, jusqu'en 1780 environ, ont reconnu la dette que la Grèce doit à l'Égypte pour ses dieux et pour sa 
culture. À la suite de la construction du « modèle aryen », par l'action conjuguée du romantisme, du positivisme, du christianisme et de la franc-maçonnerie, ce modèle ancien ne pouvait plus être accepté. Ce sont ces mouvements qui, à l'époque moderne et contemporaine, ont favorisé l'éveil du racisme, de l'idée de progrès et du " professionnalisme » dans la culture occidentale et dans les spéculations intellectuelles, allant jusqu'à masquer l'ancienne façon de penser et à imposer une nouvelle présentation, idéologique, de l'histoire et des sciences humaines. Ainsi la recherche " scientifique » consacre l'exemplarité du « miracle grec », et la Grèce est célébrée en tant que berceau de la pensée occidentale.

Trois contributions sont dues au responsable de la publication, Wim M. J. Van Binsbergen, et trois autres s'attachent à contester les conceptions de Bernal. Josine $\mathrm{H}$. Blok, historienne spécialiste du monde ancien et moderne, insiste sur la méthodologie historiographique et sur les connaissances que, au début du $\mathrm{XX}^{\mathrm{e}}$ siècle, détenaient les savants tant critiqués par Bernal. Elle s'appuie sur le « cas » de Karl Otfried Müller, le linguiste et historien allemand, présenté comme un fervent défenseur des Grecs, insensible à la fascination qu'exercent les Égyptiens, et principal responsable du changement de conception de l'histoire grecque au profit du « modèle aryen ». La correspondance de Müller, inconnue de Bernal parce qu'elle fut éditée seulement dans sa langue d'origine, l'allemand, prouve à quel point les accusations de racisme, d'antisémitisme et de romantisme adressées à son encontre sont infondées. Un jugement qui vise à condamner les acteurs de l'histoire devrait être soumis à un examen sérieux de toutes les informations existantes, ainsi qu'à l'analyse des facteurs sociaux et politiques externes pesant sur l'histoire des sciences, et à un examen attentif des méthodes et des informations provenant de l'intérieur. Des arguments aussi sensibles que ceux tournant autour des concepts de race, d'ethnicité, d'identité nationale... réclament une parfaite honnêteté politique et scientifique.

L'archéologue et linguiste Jan Best étudie les toponymes anciens de la ville de Mellia, en Crète, en s'appuyant sur le modèle d'une lecture posteurocentrique de documents datant de l'âge du bronze. Les thèses de Bernal sont utiles pour mener des études microscopiques, comme celles touchant les sceaux crétois datant du II ${ }^{e}$ millénaire. $S^{\prime}$ ils montrent la transmission des techniques égyptiennes, ils témoignent également de l'existence de relations avec l'Anatolie, la Syrie, et la Palestine. Bernal a posé ouvertement aux non-professionnels des questions que les spécialistes connaissaient : les échanges existant dans la Méditerranée orientale ont supplanté les recherches sur les « maîtres grecs ». Il convient toutefois de ne pas substituer les Grecs aux Égyptiens ! L'égyptologue Arno Egberts rejette l'expression égyptienne $h t n t$, « temple de [la déesse égyptienne] Neith [sur le Delta] », proposée par Bernal, expression qu'il considère comme étant l'origine du nom de la divinité grecque « Athéna », s'appuyant en cela sur l'incompatibilité phonétique (consonnes en collision) et la structure lexicale inexacte. Bernal confirme l'étymon et y ajoute également celui de " parthénon », " salle des vierges » à Athènes, dérivé du toponyme égyptien $p r$ th $n$, « maison de la splendeur », dérivation aussi douteuse, nonsense pour Egberts. Toutefois, l'auteur de BA clarifie la question en ces termes : « [les deux racines et les similitudes sémantiques] ne donnent pas une étymologie certaine mais seulement très plausible, et d'autant plus qu'elles se renforcent mutuellement » (p. 171). Bravo ! À supposer des incertitudes et en progressant de proche en proche, la déduction finale arrivera... à en prouver la certitude! Quelles sont les limites d'une telle procédure simpliste que Bernal utilise souvent? Si la généralisation de cette méthode se répand, la porte semble ouverte à 
l'arbitraire. Plusieurs « chercheurs » surgiront dans chaque domaine de la science pour mettre en place leur propre savoir, des savoirs parallèles soutenus par des logiques affectives, partielles ou idéologiques. En effet, le toponyme désignait le sanctuaire d'Osiris à Saïs, la ville du delta occidental du Nil (aujourd'hui Sa el-Hagar) sise dans le même secteur que le temple dédié à la déesse égyptienne Neith. Le site est lié, nous informe Bernal, à la Libye orientale soit à cause de l'affinité du phonème égyptien avec diverses autres étymologies possibles, lesquelles indiqueraient « faïence, les yeux divins [les yeux pers d'Athéna] » et " population [libyenne], huile d'olive », soit pour des manifestations de rites connus également en Libye. De la sorte, Athéna, la déesse aux yeux pers, proviendrait d'Afrique, ou plus exactement des populations nordafricaines du Delta qui ne sont guère noires ! Elle est devenue « noire " grâce au choix de l'éditeur américain de Bernal, qui a opté pour le titre Black Athena plutôt que pour celui d'Athéna africaine, préférable, nous avoue Bernal. Évidemment, privée de «noire », la force du scoop publicitaire, dans « l'opération Bernal », aurait été moindre.

Les lecteurs de l'ouvrage pourront encore apprendre, à travers les Réponses de Bernal, que désormais les mots grecs d'origine sémitique ne forment plus le quart du vocabulaire désigné dans $B A$, mais seulement $15 \%$ ! À l'égard des langues et des cultures africaines, Bernal semble peu évoluer dans leur connaissance. Il renonce à son scepticisme initial sur une bonne partie des thèses de Cheikh Anta Diop, mais n'admet ni les «stéréotypes » répandus en Afrique occidentale et centrale, ni l'existence en Afrique d'une famille linguistique panafricaine unique. En revanche, les descendants de l'Homo sapiens sapiens de l'Afrique orientale, vieux de 100000 ans, constituent le prototype de la population mondiale non africaine et on les retrouve en outre dans les populations actuelles de la Corne de l'Afrique (somalienne, agaw et falasha).

Il accepte l'hypothèse qu'un lien entre la langue wolof du Sénégal et l'ancien égyptien pourrait exister. Le groupe linguistique Niger-Congo de Greenberg, dont le wolof fait partie, n'est pour Bernal qu'une branche du groupe plus ancien nilo-saharien, à l'intérieur du grand ensemble afro-asiatique qui rassemble le sémitique, le tchadique, l'égyptien, le berbère et les langues couchitiques. Il serait possible que des éléments des langues parlées dans le Sahara il y a 10000 ans, pendant l'holocène et la formation de la savane, soient préservés dans le wolof. De ce fait, non seulement des éléments des langues nilosahariennes, mais également des langues sahariennes, se trouveront dans les langages nubiens et dans ceux de la Haute-Égypte. Ce vaste groupe linguistique afroasiatique, soutient alors Bernal, ne s'est pas nécessairement manifesté à partir du centre de l'Afrique orientale, zone comprise entre l'Éthiopie et le Kenya ; il a pu se propager depuis le Haut-Nil, où se trouvent des locuteurs de langues nilo-sahariennes. Les Égyptiens autochtones de la Haute-Égypte étaient fondamentalement ces descendants d'Africains, mélangés avec des Centrafricains et des Africains de l'Ouest. Autrement dit, les Égyptiens de la BasseÉgypte, du Delta, étaient des Nord-Africains avec des apports d'individus venus de l'Est africain et du Sud-Ouest asiatique. Le premier écrit de Wim Van Binsbergen veut réaliser un réajustement " constructif » et un contrôle des thèses de Bernal, dix ans après qu'elles aient été émises, en s'appuyant sur des considérations théoriques. Du point de vue des transformations et de l'adaptation d'un élément culturel lors de sa diffusion, les liens que l'Athéna grecque aurait eus avec l'Égypte seront mieux compris si l'on considère l'arrière-plan égyptien et l'insertion dans l'histoire grecque. Les questions posées dans BA, selon Wim Van Binsbergen, ont ouvert une voie utile pour réaliser un programme de recherche globalement valable, quoique son développement nécessite le travail d'une équipe 
entière. Le recours à une collaboration interdisciplinaire nous semble en effet être le premier résultat direct issu de $B A$.

L'autre essai de Wim Van Binsbergen analyse les interactions de deux modèles alternatifs. Le premier modèle d'écriture crétoise désigne l'île de Crète en tant qu'unique point géographique où se sont opérées les transformations des apports intercontinentaux. Le deuxième modèle s'appuie sur l'exemple de Byblos et la côte Nord syrienne et du Sud-Est anatolien en tant que points locaux, séparés de la localisation évolutive des apports intercontinentaux. En conclusion, les écritures primitives de Crète révèlent des apports égyptiens, arrivés en Crète par le Levant, en même temps que d'autres emprunts originaires d'Anatolie.

Dans une dernière étude, Van Binsbergen applique une méthode comparative à une analyse historique. Deux importants systèmes formels d'Afrique -- la pratique divinatoire employée dans la géomancie et l'activité du jeu à tablettes nommé mankala -- se sont répandus dans le monde entier à partir d'une zone particulière d'Afrique au cours de la période néolithique. Ses conclusions sont globalement en accord avec les thèses de Bernal, surtout en ce qui concerne leur vérification empirique et le repérage des failles de la culture occidentale dans l'appréhension du continent noir. On regrettera que dans ce volume certains aspects de l'afrocentrisme, chers à Bernal, n'aient malheureusement pas fait l'objet d'un exposé spécifique.

\section{NOTES}

1. M. R. Lefkowitz \& G.MacLean Rogers, eds, Black Athena Revised, Chapel Hill-London, University of North Carolina Press, 1996. Cf. le compte rendu de F.-X. Fauvelle dans Cahiers d'Études africaines, XXXIX (1), 153, 1999, pp. 207-210.

2. M. Bernal, Black Athena. Les racines afro-asiatiques de la civilisation classique, vol. I, L'invention de la Grèce antique 1785-1985, Paris, PUF, 1996, traduction du volume publié en anglais en 1987. Le deuxième tome, publié en anglais en 1991, n'est pas encore, en mai 1999, paru dans sa traduction française. Voir la critique de M. Sartre, «Grèce fille d'Afrique ? Martin Bernal défend l'idée d'une culture hellénique surtout redevable à l'Égypte et au Proche-Orient sémitique. Une thèse délaissée depuis longtemps dont la démonstration scientifique reste à faire ", Le Monde, 13 décembre 1996, p. XV. Le plan complet de l'ouvrage avait prévu quatre volumes. 